\title{
Matthäus 16, 18. \\ Laienbemerkungen zu der Untersuchung Dells, ZNW XV, 1914, 1 ff.
}

Von Otto Immisch in Freiburg i. Br.

Der Titelzusatz will durchaus als ein Ausdruck der Bescheidenheit verstanden sein. Die gesamte theologische Arbeit in einer Sache von solcher Wichtigkeit, wie sie die Grundstelle vom Primat des Petrus besitzt, kann ich unmöglich übersehen und erschöpfen. Andrerśeits erscheint mir die vorzutragende Auslegung bei immer erneutem Durchdenken haltbar und richtig, und ist sie es, so dürfte das nicht ohne weitere Bedeutung sein. Wird sie von den Theologen widerlegt, so ist mindestens ihrer Wiederholung vorgebeugt, und sollte sie gar, ohne daß ich es weiß, schon erledigt sein, so wäre die Erneuerung der Gegengründe auch deshalb von Nutzen, weil sie offenbar-nicht bekannt und zugänglich genug sind. -

Dells Ergebnis ist: die Stelle ist nicht erst geschaffen dem Papsttum zuliebe, sie gehört dem Matthäus. . Aber sie ist kein Heilandswort. Sie ist vom Evangelisten zusammengewoben aus verschiedenen Bestandteilen einer sehr alten Petruslegende, deren volkstümliche Motive Dell auf religionsgeschichtlichem Wege sorgsam aussondert: das

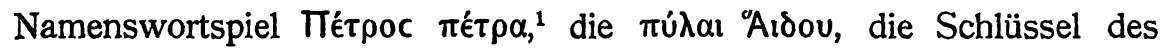
Himmelreichs, das Binden und Lösen. Wenn nun freilich S. 46 aus dieser sehr berechtigten Sonderung der Schluß gezogen wird, eine derartige Häufung verschiedenster Vorstellung in einem Ausspruch von Jesus selber bei einer bestimmten Gelegenheit sei unmöglich, so liegt eine petitio principii vor. Wer vermöchte in solchen Dingen die Grenzbestimmung des für Jesus Zulässigen bündig nachzuweisen? Die Willkür tritt schon bei den Einzelheiten hervor. Da wird S. $21 \mathrm{ff}$. recht ansprechend über etymologisches Spielen mit Eigennamen überhaupt

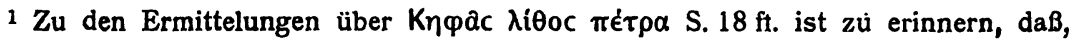
wenn die romanischen Sprachen für Stein nur petra und nicht lapis fortsetzen, dies nicht nur aufs volkstümliche Latein, sondern auch aufs volkstümliche Griechisch Schlüsse erlaubt. 
gehandelt, aber daß nun Jesus selber sich dieses so volkstümlichen Mittels - neben wievielen anderen! - nicht auch seinerseits hätte bedienen können, dafür kann ich den zwingenden Beweis nirgends wahrnehmen. Und ebenso steht es mit den andern Motiven. Gerade Dell zeigt ja doch ihr Alter und ihre Verbreitung: warum also hätte sich Jesus ihrer nicht bedienen dürfen, wenn er doch beispielsweise die dem Ursprung und dem Wesen nach gleichartigen Vorstellungen vom Teufel und seinem Dämonenreiche aus seiner Umwelt vorbehaltlos, wie es scheint, übernommen hat? Auszunehmen wäre in Dells

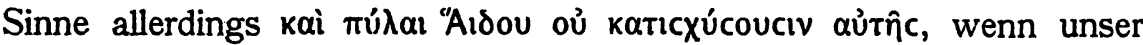
Verfasser recht hätte mit der neuerdings auch von andrer Seite aufgestellten Behauptung, hier sei bereits die Lehre von Christi Hadesfahrt vorausgesetzt (S. 31). Aber eben hiergegen wendet sich die Auslegung, die ich sachverständiger Prüfung vorlegen möchte. Nach ihr ist diese Voraussetzung durchaus entbehrlich, ja abzuweisen.

Dell beleuchtet den Ausspruch ausschließlich von seiner sozusagen generellen Seite her, von seiner Teilnahme an weitverbreiteten volkstümlichen Vorstellungen, er übersieht dagegen seine individuelle Seite, die zeitlich-örtliche Bestimmtheit der Situation, in die er gehört, das Landschaftsbild, unter dessen Eindruck nach der evangelischen $\mathrm{Er}$ zählung das Herrenwort gesprochen ist. Jesus befand sich damals in

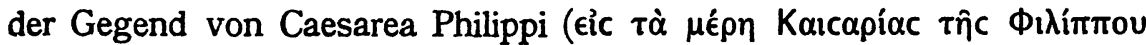

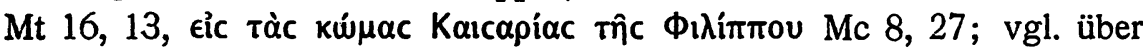
die kŵpal Schürer II $^{8} 161$ und jetzt Rostowzew, Arch. f. Pap., Beiheft I 1910, 261 f.). Es ist dieselbe malerische Landschaft, deren noch jetzt allgemein gerühmter Eindruck schon den Dichter des 41. (42.) Psalms tief ergriffen hat. Ich bin sicherlich nicht der erste, der die Örtlichkeit und den Inhalt der Petrusszene miteinander in Verbindung setzen möchte. Bekannt ist mir geworden, daß es schon G. A. Smith getan hat, worauf noch zurückzukommen sein wird (Hist. geogr. of the holy land, Lond. $1895,476 \mathrm{ff}$.). $\mathrm{DaB}$ er dabei gerade den entscheidenden Schritt nicht vollzog, nämlich die Erklärung eben des Verses 18 aus

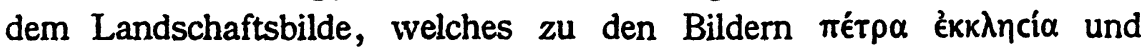
$\pi u ́ \lambda a l$ "Alठov - in diesem unmittelbaren Nebeneinander -- mit sichtbarlicher und greifbarer Wirklichkeit auf das natürlichste anregte, dies Unterlassen der nächstliegenden Erklärung vermag ich nur aus dem Verdikt $\mathrm{zu}$ begreifen, das nun einmal über das „Primatzeugnis“ in weiten theologischen Kreisen verhängt ist. Es ist freilich ziemlich folgenschwer, wenn gerade dieses Wort Heimatfarbe und Bodenständigkeit aufweist. Aber die kirchengeschichtlichen Folgerungen dürfen 
nicht maßgebend sein für die Interpretation, die sich an die Tatsache $\mathrm{zu}$ halten hat, daß die vom Evangelium selbst bezeichnete Örtlichkeit drei Stücke vereint darbot: eine ragende Felswand und darauf gegründet den leuchtenden Bau eines Heiligtums sowie in unmittelbarer Nachbarschaft dieses Baues einen schwarz gähnenden Höllenschlund.

Wegen des historischen, topographischen und archäologischen $\mathrm{Ma}-$ terials verweise ich außer auf Schürer und Smith auf Guérin, descr. géogr. hist. et arch. de la Palestine III 2, Paris 1880, $308 \mathrm{ff}$., Buhls Geogr. v. Pal. (1896) 239 f., Benzinger in Pauly-Wissowas RE III 1290 f., auch auf Neubauer, études talmudiques I, Paris 1868, $236 \mathrm{ff}$. Abbildungen der Stadt und auch der Höhle z. B. bei Ebers und Guthe, Palästina in Bild und Wort I 357. 359. Man vergleiche ferner die zum Teil illustrierten Berichte über Institutsreisen in Dalmans Palästinajahrbuch I, 1905, $90 \mathrm{ff}$. (Schwöbel) und IV, 1908, $104 \mathrm{ff}$. (Greßmann). Überdies durfte ich zusammen mit lehrreichen Photographien das noch unveröffentlichte Tagebuch benutzen, das mein Kollege $H$. Thiersch über eine im Auftrag der Deutschen Orient-Gesellschaft unternommene Palästinareise geführt hat, auf welcher er im Juni 1903 auch nach Caesarea Philippi kam. Er zuerst hat, so scheint es, für den Standort des Augustustempels bei der Höhle sichere Anhaltspunkte gewonnen.

In unmittelbarer Nähe der am Südfuß des Hermon gelegnen antiken Stadt befindet sich die Felswand, an welcher der Jordan ent-

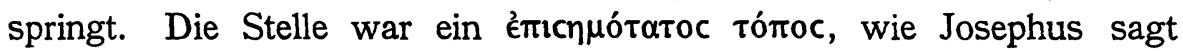
(Arch. XV 10, 3). Die Jordanquelle, das Andenken des Psalmisten, die Berühmtheit der Örtlichkeit überhaupt, das alles vereint muß es im ursprünglichen Zusammenhang der Erzählung für selbstverständlich haben erscheinen lassen, daß die Petrusszene, die ja ausdrücklich nicht in die Stadt selbst, sondern in ihre Umgebung verlegt wird, eben an dieser gefeierten Stelle gedacht ist. Auch für Smith ist das eine ganz selbstverständliche Annahme. Wer in der Erforschung volkstümlicher

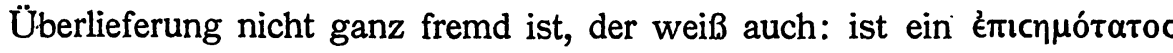

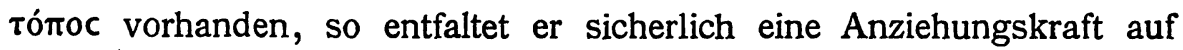
die Überlieferungen seines Umkreises.' Und hier steht es obendrein

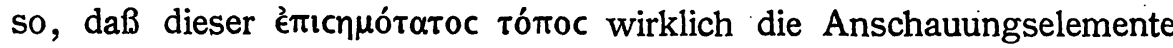
vereinigt enthält, die den Voraussetzungen der Szene entsprechen. Das darf scharf betont werden, dem etwaigen kümmerlichen Einwand gegen-

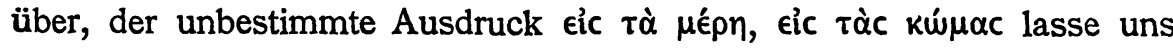
Spielraum, und die Sache könne sich ja auch an irgendeinem andern und obskuren Winkel des Gebietes abgespielt haben. Wir wollen doch

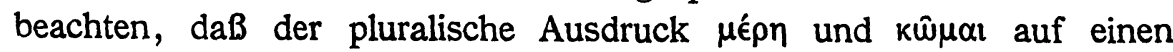


Wanderaufenthalt deutet, innerhalb dessen die natürliche Lokalisierung der Szene denn doch wohl die ist, durch welche die immer nächstliegende, und das ist eben die anschauungsmäßige Interpretation, sich ergibt. Der Gegner wird sich bei dieser Sachlage mit dem allgemeinen Vorbehalt vager Möglichkeiten nicht begnügen dürfen, er muß die angenommene Ortsbeziehung auszuschließen vermögen. Dies scheint mir die Methode zu fordern. Nun das Einzelne.

Die Höhe der Felswand wird zu 30 Meter angegeben (Ebers und Guthe), oder zu „etwa 150 Fuß" (Smith). In-ihr befindet sich, über dem etwa 30 Fuß breiten Quellbereich des Jordans, am untern Saume eine mächtige Höhle, die berühmte Pansgrotte, das חávєıov. Die Besucher berichten einstimmig, daß sie - wohl durch Erdbebeneinstürze - gegenwärtig gegen früher stark verkleinert und verschüttet sich darstellt. Auch ist sie jetzt wasserlos, wenn auch zuzeiten ein schmutziger Tümpel darin vorgefunden wurde. Doch ist „in der Rückwand ein alter Quellspalt zu erkennen“, aber „der Boden ist ganz geschlossen und voll großer Trümmer" (Thiersch). Ganz anders stand es mit der Höhle und der Jordanquelle zu Josephus' Zeit. Da kam man durch die Höhle an eine für unergründlich geltende Abgrundtiefe, aus deren Wasser die Flußquelle draußen herstammen sollte. Bell. I 21, 3: ěv $\theta \alpha$

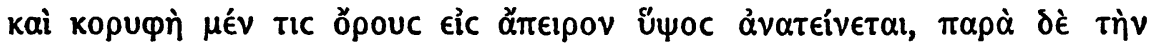

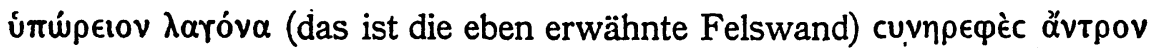

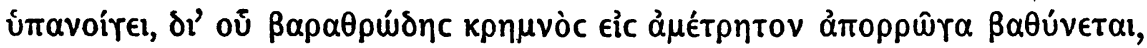

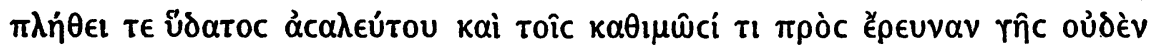

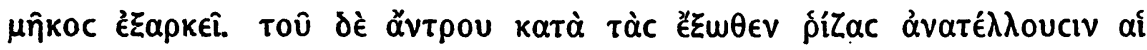

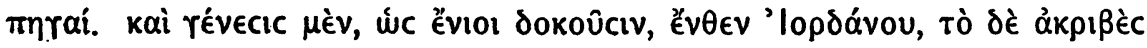

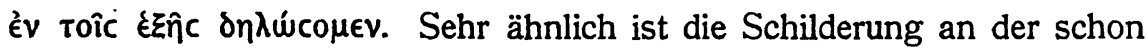
erwähnten Stelle Arch. XV 10, 3. Die Verweisung auf $\tau \dot{\alpha}$ Ė $\xi \hat{c}$ geht auf III 10, 7, wo wir erfahren, die eigentliche Jordanquelle sei der nicht sehr fern gelegne runde Phialasee (Birket-rān, abgebildet Pal. Jahrb. I, Taf. 4, 3), der angeblich, nach einem vom Tetrarchen Philipp angestellten Versuche, in unterirdischer Verbindung mit. der Panshöhle stehen soll. Diese Sache kann auf sich beruhen, ${ }^{1}$ man sieht aber, der

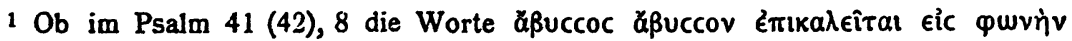

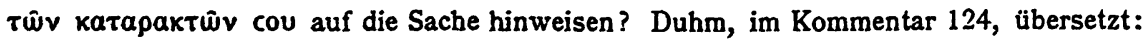
„Flut ruft zu Flut beim Donner deiner Katarakte“, denkt aber an die in Bergschluchten herabstürzenden herbstlichen Regengüsse. Seine Erklärung berücksichtigt das Besondre der Landschaft leider überhaupt nicht ausreichend. Bezeichnend ist, daB auch auf diesen Dichter die Felswand ihren Eindruck nicht verfehit. V. 10: „Sprechen muß ich zu Gott meinem Fels" (eigentlich: meines Felsens, in LXX verwischt). 


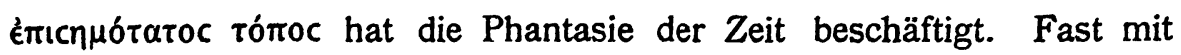
Naturnotwendigkeit mußte eine Höhle dieser Art auch zu religiösen Vorstellungen Anlaß geben, und zwar sicherlich $\mathrm{zu}$ solchen, die wir kurz und unmißverständlich mit dem Worte "chthonisch" bezeichnen können. Der griechische Pan, der dem Orte den Namen gab und dessen Kult auch Inschriften und Münzen bezeugen, ist dafür natürlich nicht ohne weiteres maßgebend. Freilich können wir ihn weit zurückverfolgen. Panion hieß der Ort schon, als 198 Antiochus d. Gr. seinen entscheidenden Sieg über die Ägypter dort gewann (Polybius XVI, 18, 2 und XXVIII, 1, 3. Aus der erstgenannten Stelle darf man übrigens schließen, daß die Landschaft bereits den getadelten Historiker Zenon zu einer rhetorischen Ekphrasis reizte, also schon damals ein

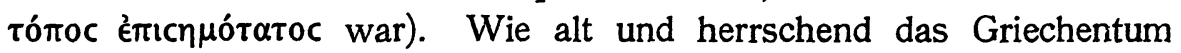
und sein Kult an dieser Stelle war, zeigt auch der Name der Stadt. Nachdem sie im Jahr 20 durch Augustus an Herodes gekommen war, ging sie von diesem an seinen Sohn Philipp über, der sie (3 oder 2 v. Chr., wie es scheint) zu Caesarea umtaufte (was den Unterscheidungsnamen $\Phi_{i} \lambda i$ intou nach sich zog, während der Talmud Kisrion; etwra soviel wie Klein-Caesarea kennt). Aber der amtlichen Willkür zum Trotz, die sich noch erfolgloser erwies, als Agrippa II. aus der Stadt eine Neronias machte, tut ein Überblick über alle Zeugnisse dar, daß der alte mit dem Kult verknüpfte Name Mavióc oder Mave(i)ác, manchmal in Verbindung mit Caesarea, sich erhielt und schließlich so durchsetzte, daß der Ort noch heute Bānijās heißt (vgl. z. B. Eus., Hist.

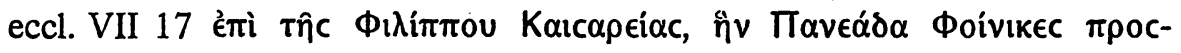
aropeúoucı). Es kann auffallen, daß das volkstümliche Evangelium überhaupt der amtlichen Bezeichnung sich bedient, vielleicht doch aus Scheu vor dem heidnischen Kultnamen; vgl., was sich aus der von Josephus Vita 13 erzählten Geschichte über die gegen die Griechen abgeschlossne Art der Juden von Panias sich ergibt.

Über Art und Wesen des Panskultus von Caesarea gestattet die spärliche literarische, epigraphische und monumentale Überlieferung kein hinreichend sicheres Urteil. Wir können uns keineswegs z. B. an. das konventionell-idyllische Münzbild halten, das den Gott an einen Baum gelehnt zeigt, flötenblasend. Man müßte wissen, ${ }_{i s}$ welcher einheimischen Gottheit Nachfolger er war und welche Vorstellungen die Nichtgriechen mit ihm verbanden. Freilich, auf die Vermutungen über die vorhellenischen Kulte der Gegend einzugehen, führt zu nichts. Sie sind zurzeit ebensowenig geklärt, wie die gleichfalls von altersher nur vermutungsweise und zum Teil sicher falsch beantwortete Frage, wie 
die vorhellenische Stadt hieß, die dort lag. Ebensowenig läßt sich von der entgegengesetzten Seite her der. Schleier lüften. Es steht - nämlich heute ein wenig links oberhalb der Panshöhle ein Heiligtum des auch vom Islam verehrten St. Georg, mit seinem Grabe drin. Aber in das. Wirrsal der St. Georgs-Forschung werden wir uns um so weniger begeben, als keinerlei historische und archäologische Daten über diesen Bau erforscht zu sein scheinen und also selbst die Voraussetzung irgendwelcher Kultkontinuität vor der Hand in der Luft schwebt. Wir bedürfen auch aller dieser Hilfen nicht, denn wir besitzen wenigstens ein vollgültiges Zeugnis, das uns deutlich hinter die konventionelle Typik des griechischen Höhlenherrn blicken läßt und seinen nach all den landschaftlichen Voraussetzungen, wie schon bemerkt, geradezu selbstverständlichen chthonischen Charakter vollauf erweist, ich meine das von Eusebius (Hist. eccl. VII 17) erzählte Mirakel. Neben der Jordanquelle wurde an einem bestimmten Festtag ein Opfertier hingelegt, und dieses pflegte alsdann zu verschwinden,

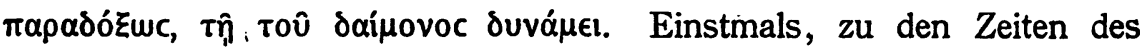
Gallienus, war zufällig der römische Senator Astyrius zugegen. Den Christen erbarmte es des heidnischen Aberglaubens. Er betete, der

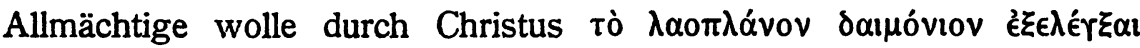

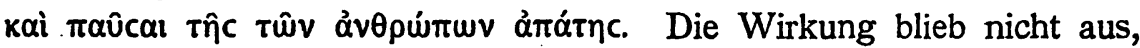
das schon verschwundene Opfertier schwamm auf einmal oben auf dem Wasser, mit dem Wunder war es für immer vorbei (also auch mit der Macht des heidnischen Gottes). Es ist klar, daß dieser Dämon - und wer anders als Pan, der Herr der Höhle, könnte gemeint sein? - in der Tiefe haust. Da hinunter zieht er das Opfer, von da gibt er es zurück. ${ }^{1}$ Das Opfer vergleicht sich seiner Art nach am besten den von Stengel, Griechische Kultusaltertümer S.121 aufgezählten: so gilt ein an der syrakusanischen Kyanequelle dargebrachtes und in sie versenktes Stieropfer der in die Erdentiefe entführten Kore (Diodor IV 23, 4), die eben an der Stelle, wo später die ihr heilige Quelle entsprang, in die Unterwelt eingezogen war (ebd. V 4).

Der chthonische Charakter des Herrn der Höhle scheint mithin sicher. Daß es überdies gerade die Pansgestalt ist, die sich unter orientalischem Einfluß allmählich in die mittelalterliche Teufelsfigur umgesetzt hat, daran sei nur nebenher erinnert. Es soll das in keiner-

1 So ganz klar nach dem Text des Eusebius. Unsinnig sind die Zusätze bei Rufinus: et erat hoc famosissimi miraculi genus, quo persuaderi adstantibus videretur in caelum hostiam invisibiliter esse sublatum, und weiterhin: victima quae invisibiliter credebatu, adsumpia. 
lei Weise betont werden. Die Art, wie ein solcher Höhlendämon auf die Volksphantasie wirken muß, ergibt sich ja von selber. Und diese Höhle von Caesarea, mit der geheimnisvollen und unergründlichen Erdspalte darin, kann es irgendwie zweifelhaft sein, daß sich die Vorstellung eines Unterweltseingangs damit verbinden mußte? $\mathrm{Da}$ haben wir denn also wirklich die $\pi u ́ \lambda \alpha \imath$ "Alठou! Zwar ist hinreichend bekannt, daß dieser Ausdruck im Bibelgriechischen (wie auch außerhalb desselben; vgl.Usener, Kl. SchriftenIV $226 \mathrm{ff}$.) nicht immer in einer noch lebensvollen Bildlichkeit gebraucht wird, sondern auch schon verblaßt und erstarrt als ein konventioneller Schmuckausdruck für "Tod" und "Todesgefahr" schlechthin. Indessen, wer solchen Ausdruck in Angesicht eines wirklichen Höllenrachens verwendet, dem wird sicher das ursprüngliche Bild im Augenblick des Sprechens wieder lebendig, zumal es seine etymologische Durchsichtigkeit niemals einbüßen konnte.

Den Felsen also und die $\pi u ́ \lambda \alpha l$ "Aloov kennen wir nun genauer.

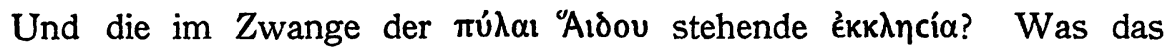
Herrenwort hier unter ékk $\lambda$ ncía eigentlich versteht, das vermag in scharfer Begriffs bestimmung wohl niemand mehr ganz sicher auszumachen, daß aber unter den sehr verschiedenartigen Anschauungsformen für diesen schwer $z \dot{u}$ fassenden Begriff, die wir kennen (vgl. Dell 30), an unserer Stelle die eines Gebäudes allein in Frage kommt, geht aus dem Verbum oikodouńcw klar hervor. Und ein heiliges Gebäude sah Jesus damals wirklich vor Augen, unmittelbar über dem dunklen Todestor, durch seine Lage gleichsam „in der Banngewalt des Reiches der Tiefe". Diese Auffassung von katıcxúcoucıv bedeutet nicht etwa eine Zurechtrückung zugunsten unsrer Auslegung. Dell hat.S. 29 vortrefflich aus Dieterichs Abraxas die Parallelen angeführt, aus denen das Rituelle des Ausdrucks im Sinne des

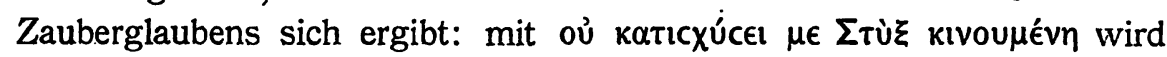

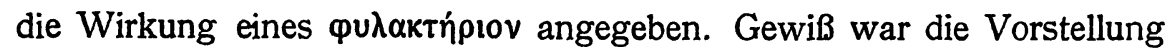
einer solchen Bannkraft der dunklen Mächte der Tiefe nahegelegt, wenn wie in unserm Falle der schwarze Höllenrachen gleichsam im Fundamente eines mit feindlichen Augen betrachteten Tempels gähnte. Und welchen Tempel konnte ein jüdisches Auge feindlicher betrachten als einen Tempel des Kaiserkultes, ein Augusteum?

Während Guérin nichts über seine Stelle an der Felsenhöhe auszumachen wußte und Smith nur vermutungsweise an die Georgskapelle als an seine Nachfolgerin denkt, ergab Thierschs Durchforschung der Baugrundmöglichkeiten und der vorhandenen Spuren als sehr wahrscheinlich, daß der Tempel etwas unterhalb der Kapelle stand, wo oben 
auf dem Felsrücken, nahe dem senkrechten Absturz und nur wenig westlicher als die Grotte unten, die Stadt beherrschend ein der Größe nach geeigneter Platz sich findet, zum Teil mit Substruktionen aufgemauert, von denen Reste erhalten sind. Als Rückwand diente stellenweise der abgearbeitete Fels, an welchem noch die Löcher sich zeigen, die der Befestigung des Marmorbelags dienen konnten. Auch sind noch deutlich die NO-Ecke und die Auflagerflächen daselbst für die Mauerquadern (ob die von Guérin S. 314 erwähnten korinthischen Säulenreste im Hofe der Kapelle zugehörig sind?). Der Tempel ward von Herodes im Jahre 20 errichtet, als er das Gebiet des Panion er-

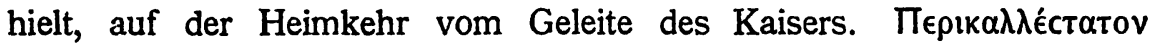

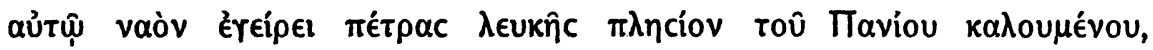
Ant. XV, 10, 3. An einer zweiten Stelle, Bell. I 21, 3, nennt Josephus weißen Marmor (vielleicht in der Form eines Plattenbelags) und spricht außer von dem hohen drüber ansteigenden Berge wiederum auch von der ungemessenen Tiefe des unmittelbar benachbarten $\beta \alpha \rho \alpha \theta \omega \dot{\delta}\rangle \eta c$

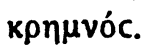

Es ward schon erwähnt, daß bereits Smith nicht verfehlt hat, den Eindruck dieses Bildes auf Jesus sich zu vergegenwärtigen (S. 476). Er legt zunächst den Nachdruck auf das Nebeneinander des Naturkultes in der Pansgrotte und der Verehrung des Staatsoberhauptes im Augusteum. Weiterhin aber betont er ganz besonders die Wirkung des Kaiserkultmales. Clear confession of Christ's divine sonship was made near the shrine in which men already worshipped a fellowman as God (S. 478). Ein sichtbarliches Sichgegenübertreten der zwei Religionen centred in individuals! Beide befriedigen die Sehnsucht for some embodiment of authority. Und doch wie verschieden beide! $\mathrm{Da}$ ein im Flusse des geschichtlichen Auf und Nieder stehender zeitlicher Machtfaktor, auf der andern Seite das verkörperte Ewige.

Wie berechtigt diese Auffassung ist, liegt auf der Hand. Wir sehen in diese Dinge heute noch viel tiefer hinein, nachdem besonders von Deißmann (Licht von Osten ${ }^{2} 225$ ff.) so erfolgreich die „Kontraststimmung" erläutert worden ist, die der Kaiserkultus auslöste. Selbst die Sprache des Neuen Testaments durchdringt sie weithin, indem mit unverkennbarer Absichtlichkeit Ausdrücke und Wendungen auf den Christuskult übertragen werden, die ursprünglich geprägt waren, um den Fürsten dieser Welt und was zum Umkreis seiner Macht und seiner Ehre gehört $\mathbf{z u}$ bezeichnen. Diese Stimmung in ihren Anfängen schon in einem Herrenwort zu finden, kann nicht befremden. 
Wir vervollständigen demnach zuversichtlich die von Smith begonnene Auslegung für unsern v. 18. Eine Pharaphrase ist dabei wohl das zweckmäßigste.

Mit Handbewegungen, die nacheinander auf Fels und Tempel und Höhle deuten, spricht der Heiland zum Jünger: Du bist der Felsenmann, ein Fels, so hoch und fest wie dieser da. Auf dich, meinen Felsen, werde ich meine Ekklesia bauen. Sie wird auf dir noch sicherer gegründet stehen wie auf seinem Felsen der Heidenbau da oben zu Ehren des weltlichen Herren, der nun schon der Sterblichkeit seinen Zoll gezahlt hat. Sichtbarlich steht ja auch sein Tempel in der Banngewalt des Todesreichs, das schwarz dort unter ihm emporgähnt.

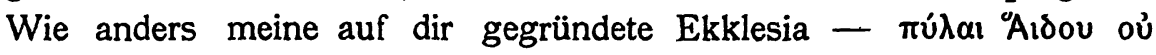
katıcxúcoucıv aủrîc. ${ }^{1}$

1 Nur anmerkungsweise erwähne ich die Vorstellungen im Hermas Pastor, in denen doch wohl alte Elemente stecken könnten. Parab. 9 zeigt auf der $\pi \varepsilon \dot{\tau} \rho \alpha$ Chri-

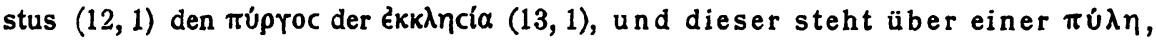

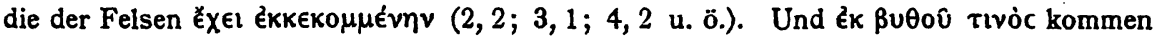

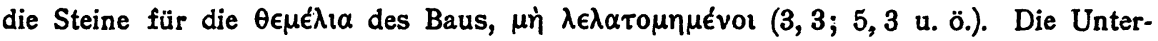

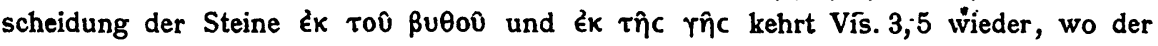
Turm - Ėi $\delta \delta a ́ \tau \omega v$ erbaut wird! Die Fülle der Erweiterungen und Allegorien sind eine Sache für sich. Aber ist es so undenkbar, daß all diesem Gewirr als Elemente die wirklichen landschaftlichen Voraussetzungen des Herrenwortes von der Ekklesia letzten Endes zugrunde liegen? 